\title{
Parkinsonian Beta Dynamics during Rest and Movement in the Dorsal Pallidum and Subthalamic Nucleus
}

\author{
-Robert S. Eisinger, ${ }^{1}$ - Jackson N. Cagle, ${ }^{2}$ Enrico Opri, ${ }^{2}{ }^{-}$Jose Alcantara, ${ }^{2}$ Stephanie Cernera, ${ }^{2}$ Kelly D. Foote, ${ }^{3}$ \\ Michael S. Okun, ${ }^{1,3,4}$ and ${ }^{-}$Aysegul Gunduz ${ }^{1,2}$ \\ ${ }^{1}$ Department of Neuroscience, Norman Fixel Institute for Neurological Diseases, University of Florida, Gainesville, Florida 32611, 22. Crayton Pruitt \\ Department of Biomedical Engineering, University of Florida, Gainesville, Florida 32611, ${ }^{3}$ Department of Neurosurgery, and ${ }^{4}$ Department of Neurology, \\ Norman Fixel Institute for Neurological Diseases, University of Florida, Gainesville, Florida 32611
}

In Parkinson's disease (PD), pathologically high levels of beta activity $(12-30 \mathrm{~Hz})$ reflect specific symptomatology and normalize with pharmacological or surgical intervention. Although beta characterization in the subthalamic nucleus (STN) of PD patients undergoing deep brain stimulation (DBS) has now been translated into adaptive DBS paradigms, a limited number of studies have characterized beta power in the globus pallidus internus (GPi), an equally effective DBS target. Our objective was to compare beta power in the STN and GPi during rest and movement in people with PD undergoing DBS. Thirty-seven human female and male participants completed a simple behavioral experiment consisting of periods of rest and button presses, leading to local field potential recordings from 19 (15 participants) STN and 26 (22 participants) GPi nuclei. We examined overall beta power as well as beta time-domain dynamics (i.e., beta bursts). We found higher beta power during rest and movement in the GPi, which also had more beta desynchronization during movement. Beta power was positively associated with bradykinesia and rigidity severity; however, these clinical associations were present only in the GPi cohort. With regards to beta dynamics, bursts were similar in duration and frequency in the GPi and STN, but GPi bursts were stronger and correlated to bradykinesia-rigidity severity. Beta dynamics therefore differ across basal ganglia nuclei. Relative to the STN, beta power in the GPi may be readily detected, modulates more with movement, and relates more to clinical impairment. Together, this could point to the GPi as a potentially effective target for beta-based adaptive DBS.

Key words: Parkinson's disease; deep brain stimulation; beta power; beta burst; subthalamic nucleus; globus pallidus internus

Significance Statement

It is known that subthalamic nucleus (STN) beta activity is linked to symptom severity in Parkinson's disease (PD), but few studies have characterized beta activity in the globus pallidus internus (GPi), another effective target for deep brain stimulation (DBS). We compared beta power in the STN and GPi during rest and movement in 37 people with PD undergoing DBS. We found that beta dynamics differed across basal ganglia nuclei. Our results show that, relative to the STN, beta power in the GPi may be readily detected, modulates more with movement, and relates more to clinical impairment. Together, this could point to the GPi as a potentially effective target for beta-based adaptive DBS.

\section{Introduction}

Over the years, an expansive body of literature has linked betafrequency $(12-30 \mathrm{~Hz})$ power to movement initiation and sup-

Received Aug. 25, 2019; revised Feb. 16, 2020; accepted Feb. 19, 2020.

Author contributions: R.S.E., J.N.C., E.O., J.A., S.C., M.S.O., and A.G. designed research; R.S.E. and K.D.F. performed research; R.S.E. analyzed data; R.S.E. wrote the first draft of the paper; R.S.E., J.N.C., E.O., J.A., S.C., K.D.F., M.S.O., and A.G. edited the paper; J.A. contributed unpublished reagents/analytic tools.

This work was supported by the Norman Fixel Institute for Neurological Disorders, the University of Florida Pruitt Family Endowed Faculty Fellowship, and National Institutes of Health/National Center for Advancing Translational Sciences Clinical and Translational Science Awards UL1TR001427, KL2TR001429, and TL1TR001428 to the University of Florida. This work was also supported by National Institutes of Health National Institute of Neurological Disorders and Stroke Award F30NS111841. The content is solely the responsibility of the authors and does not necessarily represent the official views of the National Institutes of Health. We thank the participants included in this research; the many staff members, clinicians, and students at the University of Florida and in the Brain Mapping Laboratory pression throughout the basal ganglia (Kühn et al., 2004; Hsu et al., 2012). In Parkinson's disease (PD), high beta activity reflects specific symptomatology, especially bradykinesia, and attenuates with pharmacological or surgical intervention (Silberstein et al., 2003; Kühn et al., 2006, 2009; Ray et al., 2008; Weinberger et al.,

that helped us accomplish this research, especially T. Wyckoff, P. Martin, C. Jacobson, S. Vaziri, A. Alvarado, W. Carlton, C. Reddy, L. Almeida, A. Ramirez-Zamora, A. Shukla, A. Patterson, W. Deeb, M. Burns, A. Elkouzi, C. Spears, J. Legacy, B. Patel, S. Chiu, Josh Wong, K. Moore, K. Au, J. Lanese, E.P. Torres, A. Le, B. Parks, S. Long, A. Cerquera, and R. Molina; and the National Center for Adaptive Neurotechnologies and P. Brunner for technical assistance.

The authors declare no competing financial interests.

Correspondence should be addressed to Aysegul Gunduz at agunduz@bme.ufl.edu.

https://doi.org/10.1523/JNEUROSCI.2113-19.2020

Copyright $\odot 2020$ the authors 
2012; Little et al., 2013; Tinkhauser et al., 2017a). Basal ganglia beta power can be measured with local field potential (LFP) recordings in individuals undergoing subthalamic nucleus (STN) or globus pallidus internus (GPi) deep brain stimulation (DBS). At least a decade's worth of research demonstrates that beta power can be leveraged for not only exploring the pathology of $\mathrm{PD}$, but also for developing novel adaptive DBS (aDBS) paradigms that aim to decrease pathological STN beta activity. Such therapies have proven preliminarily successful and potentially better than conventional therapies (Little et al., 2013, 2016a,b; Rosa et al., 2015; Piña-Fuentes et al., 2017; Tinkhauser et al., 2017a; Velisar et al., 2019). Therefore, this line of work calls for increased attention. Although the vast majority of beta characterization has focused on the STN with aDBS, GPi implantation is increasingly accepted as an equally effective DBS target for PD (Anderson et al., 2005; Okun et al., 2009; Follett et al., 2010; Ramirez-Zamora and Ostrem, 2018). To determine whether GPi beta power can be similarly translated to patient-specific adaptive stimulation, adequate characterization and its clinical utility first need to be investigated.

To analyze beta activity, power is typically averaged across time. However, it is now known that beta activity has functionally relevant temporal dynamics. In PD, beta power is not constantly elevated; it can surpass pathological levels and return to physiological levels in a fraction of a second (Shin et al., 2017; Deffains et al., 2018). These so-called beta bursts are linked to slowing of movements (Gilbertson et al., 2005; Leventhal et al., 2012; Shin et al., 2017). Longer bursts reflect clinical impairment and reduce the speed of upcoming movements (Torrecillos et al., 2018), whereas levodopa shifts bursts from longer to shorter durations (Tinkhauser et al., 2017b). Additionally, similar to overall beta power, STN bursts become less frequent, shorter, and smaller in amplitude during movement (Tinkhauser et al., 2017b; Lofredi et al., 2019). STN bursts are also associated with bursts in a wider basal ganglia cortical network (Feingold et al., 2015; Tinkhauser et al., 2018). However, a surprisingly limited number of studies have characterized GPi beta bursts (Piña-Fuentes et al., 2017; Wang et al., 2018). To address these shortcomings, we compared STN and GPi beta power during rest and movement in people with PD. Our hypothesis was that beta dynamics, and their relationship to clinical impairment, would be similar within the STN and GPi.

\section{Materials and Methods}

Participants. We obtained LFP recordings from 19 (15 participants) STN and 26 (22 participants) GPi nuclei (Table 1). Inclusion criteria were adults with PD diagnosed by a movement disorders neurologist and undergoing unilateral STN or GPi DBS. Target selection was based on a standard interdisciplinary screening process (Okun et al., 2018; RamirezZamora and Ostrem, 2018). Participants were 66.2 $\pm 10.2($ mean \pm SD) years old. We approached potential participants during preoperative outpatient appointments $1 \mathrm{~d}$ before surgery. Following informed consent, participants completed a mock run of the behavioral task to become familiar with the experiment. Participants were encouraged to withdraw voluntary participation at any point during the experiment if they became uncomfortable or decided they no longer wanted to proceed. One participant was withdrawn due to intraoperative complications unrelated to research, and 7 were withdrawn either voluntarily or by the recommendation of the surgeon due to discomfort or fatigue during surgery prior to research. Bilateral cases were staged; that is, the 4 STN participants and $4 \mathrm{GPi}$ participants (Table 1) completed the research for both a left- and right-sided DBS implantation on separate dates.

Surgery. All participants were off dopaminergic medications for at least $12 \mathrm{~h}$ before surgery. A Cosman-Robert-Wells head frame was placed, and a CT scan was obtained for coregistration to preoperative
Table 1. Patient characteristics

\begin{tabular}{|c|c|c|c|c|c|}
\hline $\begin{array}{l}\text { Subject } \\
\text { no. }\end{array}$ & Target & $\begin{array}{l}\text { Age } \\
\text { (yr) }\end{array}$ & Gender & $\begin{array}{l}\text { Off } \\
\text { UPDRS } \\
\text { Part III }^{a}\end{array}$ & Primary reason for surgery \\
\hline 1 & LGPi & 76 & M & 36 & Upper extremity tremor \\
\hline 2 & LSTN & 76 & M & 48 & Upper extremity tremor \\
\hline 3 & LGPi & 56 & M & 39 & On-medication dyskinesia \\
\hline 4 & R GPi & 59 & M & NA & Upper extremity tremor \\
\hline 5 & R STN & 68 & $\mathrm{~F}$ & NA & Upper extremity tremor \\
\hline 6 & LGPi & 70 & M & 49 & Forward leaning posture \\
\hline 7 & R GPi & 49 & M & 42 & Arm and leg motor fluctuations \\
\hline \multirow[t]{2}{*}{8} & R GPi & 46 & M & 66 & Rigidity and bradykinesia \\
\hline & LGPi & 47 & & 66 & \\
\hline 9 & R GPi & 73 & M & 26 & Dyskinesia and impaired speech \\
\hline \multirow[t]{2}{*}{10} & LSTN & 59 & M & 38 & Upper extremity tremor \\
\hline & RSTN & 59 & & 38 & \\
\hline 11 & LGPi & 59 & M & 39 & Dystonia \\
\hline 12 & LGPi & 72 & M & 9 & Lower extremity tremor \\
\hline 13 & RSTN & 67 & $\mathrm{~F}$ & 45 & Upper extremity tremor \\
\hline 14 & LGPi & 72 & M & 30 & Rigidity and bradykinesia \\
\hline 15 & LGPi & 78 & $\mathrm{~F}$ & 35 & Upper extremity tremor \\
\hline 16 & LSTN & 68 & M & 37 & Bradykinesia \\
\hline 17 & LGPi & 65 & M & 43 & Freezing \\
\hline \multirow[t]{2}{*}{18} & LSTN & 80 & M & 43 & Upper extremity tremor \\
\hline & RSTN & 81 & & 46 & \\
\hline 19 & LGPi & 71 & $\mathrm{~F}$ & 41 & Motor fluctuations \\
\hline 20 & R GPi & 81 & M & 39 & Tremor \\
\hline 21 & LSTN & 56 & $M$ & 33 & Upper extremity tremor \\
\hline 22 & R GPi & 77 & $\mathrm{~F}$ & 22 & Dyskinesia \\
\hline 23 & LGPi & 53 & M & 31 & Impulse control disorder \\
\hline \multirow[t]{2}{*}{24} & LGPi & 44 & $\mathrm{~F}$ & 55 & Bradykinesia and rigidity \\
\hline & R GPi & 44 & & 55 & \\
\hline 25 & R STN & 65 & M & 62 & Upper extremity tremor \\
\hline \multirow[t]{2}{*}{26} & LGPi & 64 & M & 29 & Freezing \\
\hline & R GPi & 64 & & 29 & \\
\hline 27 & LGPi & 77 & M & 20 & Upper extremity tremor \\
\hline 28 & LGPi & 68 & $\mathrm{~F}$ & 33 & Upper extremity tremor \\
\hline \multirow[t]{2}{*}{29} & LSTN & 60 & M & 42 & Motor fluctuations \\
\hline & R STN & 61 & & 42 & \\
\hline 30 & R STN & 65 & $\mathrm{~F}$ & 51 & Upper extremity tremor \\
\hline \multirow[t]{2}{*}{31} & R GPi & 73 & M & 34 & Upper extremity tremor \\
\hline & LGPi & 73 & & 34 & \\
\hline 32 & LSTN & 63 & M & 21 & Upper extremity tremor \\
\hline 33 & R GPi & 44 & $\mathrm{~F}$ & 32 & Motor fluctuations \\
\hline 34 & LSTN & 64 & M & 43 & Upper and lower extremity tremor \\
\hline 35 & R STN & 78 & M & 36 & Upper extremity tremor \\
\hline 36 & LSTN & 76 & M & 37 & Upper extremity tremor \\
\hline \multirow[t]{2}{*}{37} & RSTN & 73 & M & 31 & Upper extremity tremor \\
\hline & LSTN & 73 & & 31 & \\
\hline
\end{tabular}

${ }^{a}$ Total UPDRS was not significantly different in the STN and GPi groups by Student's $t$ test $\left(t_{(41)}=0.84, p=0.41\right)$.

MRI. Images were transformed into anterior commissure-posterior commissure space. A digitized Schaltenbrand-Bailey atlas was manually fitted to each patient's MRI using a 3D affine transformation without shearing, and the desired target coordinate was chosen (Sudhyadhom et al., 2012). Microelectrode recordings were used to identify nuclei along the trajectory and for evaluating somatotopy and stimulation responses. After the final target was chosen, the DBS electrode (Medtronic, 3387 for all participants, except Participants 16 and 30, who received Abbot's St. Jude Medical directional electrode, and Participant 34, who received a Boston Scientific directional electrode), was inserted followed by monopolar review to ensure appropriate voltage thresholds. Electrode positions were measured postoperatively and reverse-normalized into the Schaltenbrand-Bailey atlas (distal contact, mean \pm SE, GPi: absolute value of $X=20.20 \pm 0.14 \mathrm{~mm}, Y=0.27 \pm 0.20 \mathrm{~mm}, Z=-7.39 \pm 0.22$ $\mathrm{mm}$; STN: absolute value $X=9.70 \pm 0.22 \mathrm{~mm}, Y=-5.29 \pm 0.26 \mathrm{~mm}$, $Z=-7.25 \pm 0.27 \mathrm{~mm}$ ). 
Experimental equipment. The DBS electrode (4 channels for Medtronic, 8 for directional leads) was connected to a bioamplifier (g.tec HiAmp, Guger Technologies), and signals were sampled at $2400 \mathrm{~Hz}$. Two corkscrew electrodes were placed in the scalp for use as ground and reference. The amplifier interfaced with BCI2000 (Schalk et al., 2004) running the experimental application on a Windows 7 computer. During the experiment, participants pressed a pressure-sensor button with the hand contralateral to the implanted hemisphere. The button was connected to a custom-made microcontroller-based circuit, which was also connected to a light sensor placed on the monitor to synchronize graphics with LFPs. Button presses were triggered when pressure exceeded $\sim 300$ g over the sensor surface $\left(132.7 \mathrm{~mm}^{2}\right)$.

Experimental design and statistical analyses. The methodology and results presented here are from baseline recordings from a study (NCT02975193) of basal ganglia electrophysiology. Participants were instructed to press a button 10 times with the hand contralateral to the implanted hemisphere and then rest for $10 \mathrm{~s}$ (see Fig. 1a). Following the first rest period, participants completed a reaction time test (data not shown) followed by an additional $10 \mathrm{~s}$ rest period. All tasks were repeated twice. In a demonstration, we recommended pressing the button approximately once per second.

Signal processing was completed in MATLAB R2016b (The MathWorks), and statistical analyses were completed in R 3.5.2. Recordings from segmented contacts on the directional leads were averaged into single channels to better match the recordings obtained from full-ring electrodes, thus leading to 4 monopolar channels. For all participants, bipolar channels were then constructed by a subtraction of LFPs from each adjacent pair of electrodes, yielding 3 bipolar channels. Signals were then filtered with a $1 \mathrm{~Hz}$ high pass, $100 \mathrm{~Hz}$ low pass, and a $57-63 \mathrm{~Hz}$ stop band Butterworth with orders determined by the maximum order resulting in a stable filter determined by the $Z$ plane (see Fig. $1 b$ ). Periods of rest and button presses were isolated for each participant. To estimate power spectral density curves, mean spectrograms were computed in $250 \mathrm{~ms}$ (ms) blocks with $200 \mathrm{~ms}$ overlap, deriving the mean power across time in each frequency from 1 to $50 \mathrm{~Hz}$ in $1 \mathrm{~Hz}$ increments. Spectrograms for rest spanned the entire rest duration, totaling four spectrograms (see Behavioral task) each $\sim 10 \mathrm{~s}$ long, and spectrograms for button presses were constructed using signals $500 \mathrm{~ms}$ before and after each button press, resulting in 20 (see Behavioral task) spectrograms, each with a duration of $1 \mathrm{~s}$. We computed the $\log _{10}$ of spectrogram output $(\mu \mathrm{V})$ to derive units of $\mathrm{dB}(\mu \mathrm{V})$. While we use $\mathrm{dB}$ units throughout, it should be noted that 0 $\mathrm{dB}$ is equivalent to $1 \mu \mathrm{V}, 20 \mathrm{~dB}$ is equivalent to $10 \mu \mathrm{V},-20 \mathrm{~dB}$ is equivalent to $0.1 \mu \mathrm{V}$, and so on.

Functional beta power frequencies were defined for each participant as the frequency between 12 and $30 \mathrm{~Hz}$ with the greatest decrease in power between movement and rest (beta power during movement minus beta power during rest), taken from the bipolar channel with the most overall beta power decrease with movement. To demonstrate the utility of this approach, we also compared this result with beta power extracted from the frequency with the least decrease in power during movement. We elected to use functional beta power frequencies in this manner rather than conventional quantification of beta peaks because estimating a $1 / \mathrm{f}$ fit for a given spectral density curve with known physiological deviations from $1 / \mathrm{f}$ (e.g., beta increase, line noise, etc.) is prone to inaccuracy. In addition, we did not select beta frequencies using visual inspection, which has been a common approach, because this is not easily reproducible. For each participant, we also examined resting beta dynamics by identifying beta bursts from a filtered signal centered at the participantspecific beta frequency with a $4 \mathrm{~Hz}$ bandwidth (see Fig. $3 a, b$ ). Beta bursts were identified when the Hilbert transform of the beta-filtered signal surpassed and returned to a percentile threshold. Beta bursts $<100 \mathrm{~ms}$ were discarded to avoid including spurious changes in beta power (Tinkhauser et al., 2017b). Metrics extracted from beta burst computations included the number of bursts per second, duration, and strength, defined as the amplitude of the burst normalized (i.e., subtraction) to the mean Hilbert power between the 50th percentile and threshold percentile. We elected to use this approach so that we could quantify the distance (i.e., severity) between "physiological" (i.e., between the 50th percentile and the defined burst threshold percentile) and "pathological" increases (i.e., above the burst threshold percentile) in beta power for each individual. Given the highly skewed distribution of beta burst strength and duration (Tinkhauser et al., 2017b), we computed the average of the median values during each resting epoch.

We compared participant-specific beta frequencies and associated power across the STN and GPi during rest, movement, and their difference using a mixed-model ANOVA, which included a random term for an effect of participants. To determine whether our findings were beta frequency-dependent, we repeated this analysis using the gamma frequency from the channel with the highest low gamma (35-45 Hz) modulation during movement. For comparisons across targets or conditions, we used paired or unpaired comparisons via Student's $t$ tests or Wilcoxon signed-rank tests for normal or non-normal data (assessed with ShapiroWilk tests), respectively. We applied one-way $t$ tests to the difference between movement and resting beta power within the GPi and STN to test for beta desynchronization.

Beta desynchronization in the basal ganglia during movement is hypothesized to relate to speed or force during movement (Singh and Bötzel, 2013; Fischer et al., 2019). We therefore additionally examined whether beta desynchronization during each button press was associated with the speed (time between start of button press to maximal pressure reached) or maximal force applied, which could be an important potential confounding factor in comparing across the GPi and STN.

Beta burst dynamics during rest were also compared across the GPi and STN cohorts. In addition, we examined the relationship between burst duration and strength at the group level and for each participant. All beta measures, including the frequency selected and the power at that frequency, were further tested for clinical relevance using correlations with preoperative, off-medication, contralateral Unified Parkinson's Disease Rating Scale (UPDRS) scores obtained during DBS evaluation using Pearson or Spearman correlations for normal or non-normal data, respectively. We required UPDRS scores to be obtained no more than 12 months before surgery. One GPi patient had scores 13 months before surgery, and 1 STN patient who already had a contralateral lead did not have an off-medication off-stimulation baseline score. There was not an additional DBS evaluation between the two surgeries for staged bilateral cases. The average duration between the surgery date and the UPDRS assessment was 5.4 months with an SE of 0.06 months. Subscore analyses from UPDRS included bradykinesia (items 23-26), rigidity (item 22), tremor (items 20-21), postural instability and gait disorder (items 2730), and bulbar (items 18-19) scores (Eisinger et al., 2019). Finally, in an exploratory analysis without $p$ value correction, we examined the relationship between beta bursts and the summed bradykinesia-rigidity score for varying burst thresholds from 55\% to $90 \%$ (increments of 5\%) (Tinkhauser et al., 2017b). We varied the burst threshold because, while bursting approaches thus far have primarily used a threshold set at the 75th percentile of the amplitude estimate of the beta-filtered signal (see Fig. $3 b$ ), this value remains arbitrary and the threshold most suitable for identifying pathological bursting is still open to investigation (Tinkhauser et al., 2017b). Univariate statistics are reported as the mean \pm SE unless otherwise noted. Significance levels were set at 0.05 .

\section{Results}

\section{Beta power during rest and movement}

Across participants, we observed considerable variability in not only beta power, but also the beta frequency with the largest power decrease (Fig. 1c-e). However, the beta frequency selected for each participant did not differ between the GPi and STN ( $p=$ 0.73 ) (Fig. $2 a$ ) and was unrelated to symptom severity (bradykinesia $p=0.67$, rigidity $p=0.50$, tremor $p=0.077$ ). In our analysis of beta power, an ANOVA revealed main effects of target $\left(F_{(1,43)}=11.2, p=0.002\right)$ and condition $\left(F_{(1,43)}=200.4, p=\right.$ $\left.10^{-15}\right)$ with a significant interaction between target and condition $\left(F_{(1,43)}=10.6, p=0.0022\right)$ (Fig. $\left.2 b\right)$. Both resting power $\left(-10.61 \pm 2.50 \mathrm{vs}-23.29 \pm 1.65 \mathrm{~dB}, t_{(41)}=4.2, p=0.0012\right)$ and movement power $\left(-20.72 \pm 2.57 \mathrm{vs}-29.63 \pm 1.57 \mathrm{~dB}, t_{(40)}=\right.$ 3.0, $p=0.005$ ) were higher in the GPi than the STN (Fig. 2b). 
a

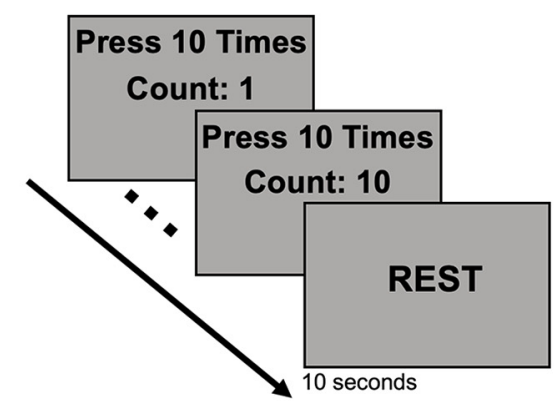

b

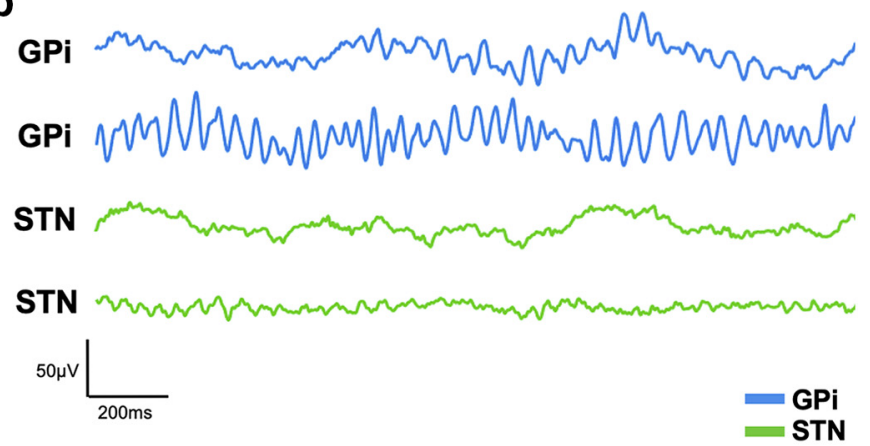

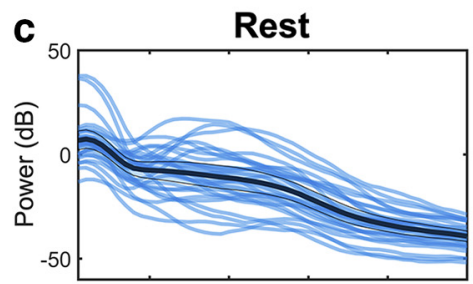
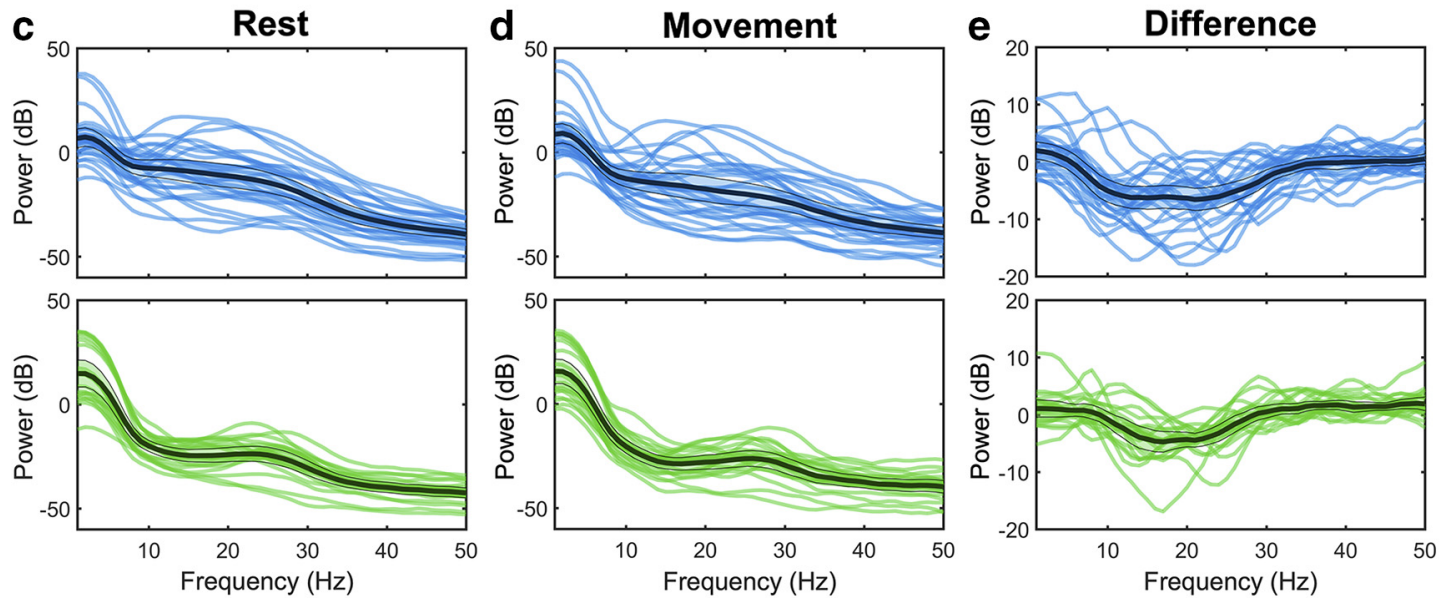

Figure 1. Variability in beta power. $\boldsymbol{a}$, Participants completed a simple task in which they pressed a button 10 times with the thumb contralateral to the implanted hemisphere while either GPi (blue) or STN (green) recordings were obtained. $\boldsymbol{b}$, Two representative filtered bipolar traces during rest are shown for each target. Power from 1 to $50 \mathrm{~Hz}$ was extracted during rest (c) and during movement ( $\boldsymbol{d}$ ) to examine differences in beta power across targets, as well as their difference (movement minus rest) (e). In each case, thick black lines indicate the group-level mean. Shaded region within the thinner black lines represents $2 \mathrm{SEs}$ above and below the mean. $0 \mathrm{~dB}$ is equivalent to $1 \mu \mathrm{V}, 20 \mathrm{~dB}$ is equivalent to $10 \mu \mathrm{V},-20 \mathrm{~dB}$ is equivalent to $0.1 \mu \mathrm{V}$, and so on

There was a significant decrease in beta power with movement in both the GPi $\left(-10.11 \pm 0.79 \mathrm{~dB}, t_{(25)}=-13.5 p=10^{-12}\right)$ and $\operatorname{STN}\left(-6.33 \pm 0.89 \mathrm{~dB}, t_{(18)}=-7.1, p=10^{-5}\right)$, but the interaction term demonstrated that there was more decrease in the GPi (Fig. 2c). This pattern was not observed for gamma frequencies (main effect of condition demonstrating higher power during movement vs rest, $F_{(1,43)}=54.5, p=10^{-8}$; main effect of target, $p=0.64$; main effect of condition, $p=0.21$ ).

Beta desynchronization did not correlate with the force applied during button presses $(p=0.77)$. More beta desynchronization was very weakly associated with faster button pressing speed $\left(R^{2}=0.015, p=0.00085\right)$, although average speed was not different for GPi and STN participants $(p=0.77)$.

As an aside, we also assessed whether the observation of more GPi versus STN beta power depended on our method of selecting beta frequencies. When computing beta power during rest and movement using the frequency associated with the least (as opposed to the most) movement-induced beta desynchronization $(24.88 \pm 1.31 \mathrm{~Hz}$ in the GPi and $24.79 \pm 1.61 \mathrm{~Hz}$ in the STN $)$, as expected there was no longer an observable decrease in beta power from rest to movement in the GPi $(p=0.12)$ or STN $(p=$ $0.99)$. Importantly, beta power during rest $(-16.79 \pm 2.77$ vs $\left.-27.06 \pm 1.99 \mathrm{~dB}, t_{(42)}=3.0, p=0.0022\right)$ and during movement $\left(-17.59 \pm 2.96\right.$ vs $\left.-24.67 \pm 2.14 \mathrm{~dB}, t_{(42)}=1.9, p=0.030\right)$ remained higher in the GPi than the STN.

Next, we related beta power to clinical impairment. In the $\mathrm{GPi}$, we observed a positive relationship between resting beta power and bradykinesia $\left(R^{2}=0.17, p=0.043\right)$, but there was only a trending relationship with rigidity $\left(R^{2}=0.15, p=0.055\right)$ and no relationship with tremor $(p=0.68)$ (Fig. $2 d$; Table 2). Beta power in the GPi during movement similarly correlated with only bradykinesia $\left(R^{2}=0.24, p=0.014\right)$ and rigidity $\left(R^{2}=0.17\right.$, $p=0.039)$ but not tremor $(p=0.52)$. We also found a correlation between total UPDRS and beta power in the GPi during rest $\left(R^{2}=0.25, p=0.012\right)$ and movement $\left(R^{2}=0.32, p=0.0030\right)$. There were no significant correlations between beta power in the STN and UPDRS subscores or total score (Table 2). The difference in beta power during rest and movement in both the GPi and STN also did not correlate with UPDRS (Table 2). There was no relationship between postural instability and gait disorder or bulbar symptoms and beta power during rest, movement, or their difference in the GPi or STN.

\section{Beta bursts}

The number of beta bursts per unit time did not differ between the GPi and STN recordings $\left(0.96 \pm 0.03\right.$ vs $1.00 \pm 0.02 \mathrm{~s}^{-1}, p=$ $0.29)$, nor did the average duration $(0.21 \pm 0.01$ vs $0.20 \pm 0.01 \mathrm{~s}$, $p=0.30$ ) (Fig. $3 c, d$ ). However, the strength of GPi bursts were higher than STN bursts $(2.41 \pm 0.26$ vs $1.07 \pm 0.11 \mu \mathrm{V}, p=$ $10^{-4}$ ) (Fig. 3e). Given the similarity in duration but not strength of beta bursts, we further examined the relationship between these variables. Across all bursts observed in this study, the strength of the burst increased on average by $0.52 \mu \mathrm{V}$ for each increase in $100 \mathrm{~ms}$ of burst duration $\left(p=10^{-15}\right)$ (Fig. $3 f$ ). At the individual level, this slope was significantly higher in the GPi compared with the STN $(0.65 \pm 0.068$ vs $0.37 \pm 0.051 \mu \mathrm{V} / \mathrm{s}, p=$ 0.0028 ) (Fig. $3 g$ ). These results suggest that, while GPi bursts are 

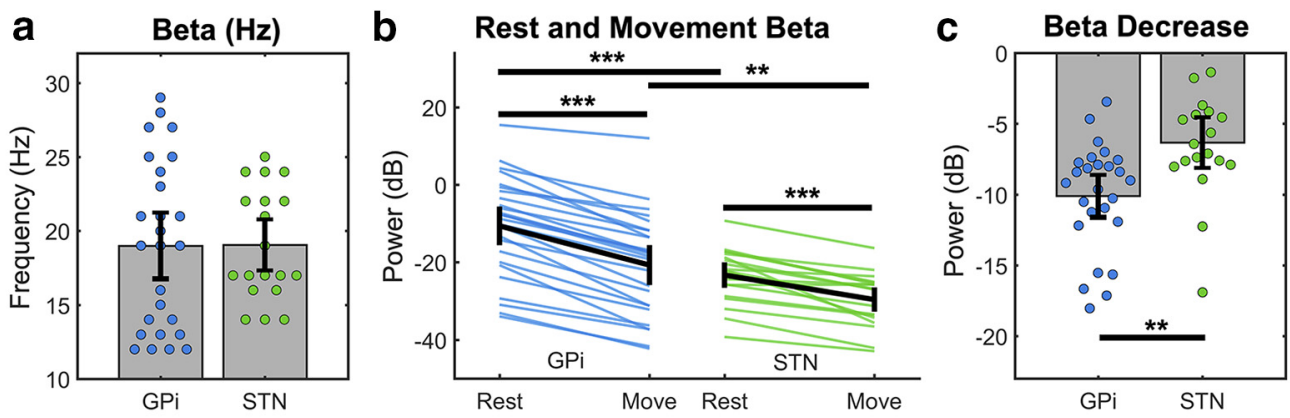

d

Bradykinesia

Rigidity

Tremor

Rest
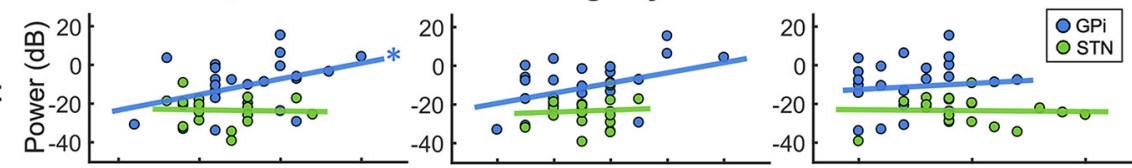

Movement
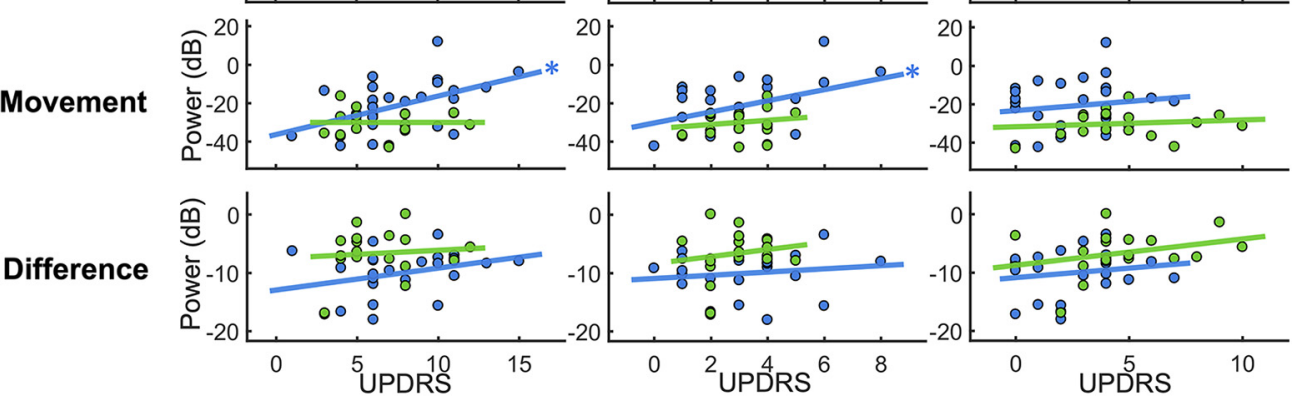

Figure 2. Beta power across targets and symptoms. $\boldsymbol{a}$, Beta frequencies were selected for each participant (see Materials and Methods) but did not differ across the GPi (blue) and STN (green). $\boldsymbol{b}$, Each blue or green line indicates the beta power at the selected beta frequency $(\boldsymbol{a})$ during rest or during movement. $\boldsymbol{c}$, The difference between rest and movement beta power is shown for each participant. $\boldsymbol{d}$, Correlations of contralateral UPDRS bradykinesia, rigidity, and tremor subscores with beta power during rest, movement, and their difference. $0 \mathrm{~dB}$ is equivalent to $1 \mu V, 20 \mathrm{~dB}$ is equivalent to $10 \mu \mathrm{V},-20 \mathrm{~dB}$ is equivalent to $0.1 \mu \mathrm{V}$, and so on. Error bars indicate two SEs above and below the mean $(\boldsymbol{a}-\boldsymbol{c}) .{ }^{*} p<0.05,{ }^{* *} p<0.01,{ }^{* * *} p<0.001$.

Table 2. Correlations between beta power and clinical scores ${ }^{a}$

\begin{tabular}{llll}
\hline Condition & UPDRS score & GPi & STN \\
\hline Rest & Bradykinesia & $R^{2}=0.17, p=0.043$ & $R^{2}=0.0024, p=0.85$ \\
& Rigidity & $R^{2}=0.15, p=0.055$ & $R^{2}=0.11, p=0.3$ \\
& Tremor & $R^{2}=0.0077, p=0.68$ & $R^{2}=0.0009, p=0.91$ \\
Movement & Total & $R^{2}=0.25, p=0.012$ & $R^{2}=0.0030, p=0.83$ \\
& Bradykinesia & $R^{2}=0.24, p=0.014$ & $R^{2}=0.0000, p=1.0$ \\
& Rigidity & $R^{2}=0.17, p=0.039$ & $R^{2}=0.030, p=0.50$ \\
& Tremor & $R^{2}=0.018, p=0.52$ & $R^{2}=0.017, p=0.61$ \\
Difference & Total & $R^{2}=0.32, p=0.0030$ & $R^{2}=0.010, p=0.69$ \\
& Bradykinesia & $R^{2}=0.10, p=0.12$ & $R^{2}=0.0091, p=0.71$ \\
& Rigidity & $R^{2}=0.038, p=0.35$ & $R^{2}=0.028, p=0.51$ \\
& Tremor & $R^{2}=0.0059, p=0.71$ & $R^{2}=0.084, p=0.24$ \\
& Total & $R^{2}=0.077, p=0.18$ & $R^{2}=0.081, p=0.25$ \\
\hline$a$ Corl
\end{tabular}

not longer than STN bursts, they tend to have a steeper durationstrength relationship.

We then examined the relationship between burst properties and key clinical symptomatology known to relate most to beta power (i.e., bradykinesia and rigidity) as we varied the threshold percentile from 55 to 90 . We did not find a robust relationship between burst duration and bradykinesia-rigidity score in the GPi (Fig. $4 a, e$ ) or STN (Fig. $4 c, g$ ) at any threshold. There was a marginally significant positive correlation between burst duration and bradykinesia-rigidity in the GPi for bursts defined by a 60 th percentile threshold $\left(R^{2}=0.09, p=0.063\right)$. We did, however, find a consistent relationship between burst strength and bradykinesia-rigidity score in the GPi (Fig. $4 f$ ) that remained significant or marginally significant at every tested threshold (e.g., at the 75th percentile, $R^{2}=0.24, p=0.012$ ), but no such relationship emerged in the STN sample (Fig. $4 d, h$ ).

\section{Discussion}

This is the first study to report differences in beta activity between the GPi and STN in PD patients during rest and movement conditions. We found higher beta power during both conditions in the GPi, which also demonstrated greater beta desynchronization during movement. This pattern was not observed for low gamma power. We also showed that beta power remained higher in the GPi, even when considering the beta frequency modulating least with movement; however, as expected at those frequencies, there was no observable movement-associated beta desynchronization. This confirms that beta modulation with movement occurs at specific frequencies but that the observation of higher beta power did not specifically stem from our methodological approach. Beta power was associated with bradykinesia-rigidity severity, however, in our data, these relationships were present only in the GPi cohort. Beta bursts were similar in duration and frequency between the GPi and STN, but the GPi showed stronger bursts, which were also correlated to bradykinesia-rigidity severity. These results overall suggest that, relative to the STN, GPi beta power may be readily detected, modulates more with movement, and may relate more to clinical impairment.

\section{Beta frequencies in the GPi and STN}

The observation that functional beta frequencies in the STN and GPi are similar supports the notion of synchrony across the basal ganglia (Brown et al., 2001). Brain oscillations at specific frequencies subserve specific motor and nonmotor behaviors (Priori et 
al., 2004; Eisinger et al., 2018). However, it is unclear why specific frequencies within an identified band vary across individuals, and what factors explain those differences (Rosanova et al., 2009; Stein and Bar-Gad, 2013). Nonetheless, we found that the frequencies selected for each participant were unrelated to clinical impairment. This is consistent with one study of STN LFPs (Kühn et al., 2009), but more work is needed, especially with recordings from multiple nuclei simultaneously.

\section{Beta characterization during rest and movement in the GPi and STN}

In $\mathrm{PD}$, elevated STN beta power during rest and its modulation with treatment have been extensively characterized (Doyle et al., 2005; Kühn et al., 2005; Wingeier et al., 2006; Engel and Fries, 2010; Giannicola et al., 2010; Yang et al., 2014; Geng et al., 2017; Piña-Fuentes et al., 2019). In contrast, fewer studies with limited sample sizes have tested whether GPi beta power is similarly elevated (Weinberger et al., 2012; Jimenez-Shahed et al., 2016; Wang et al., 2018; PiñaFuentes et al., 2019). In this study, we found important differences in beta power between these two structures.

To begin, our results suggest that the magnitude of beta power during rest and movement is larger in the GPi. There are several possible explanations for this. First, the STN participants were primarily undergoing surgery for tremor management, and scant literature has shown that tremor is associated with decreased beta power (Hirschmann et al., 2017), although as we showed, this was not true for our data. Second, signal-to-noise ratios and impedance can also affect recorded voltages. In large studies, impedance shortly after DBS implantation was similar across many targets (Wong et al., 2018). It is unlikely, though, that the tissue response would be consistently different across targets and probably adds equivalent variability to both targets in our study. Furthermore, our results are not explained by differences in impedance or signal-to-noise ratios alone, as these do not affect computations of normalized power differences, and an analysis of gamma power showed that our findings do not extend beyond the beta range. Third, the GPi has $\sim 4$ - to 5 -fold more volume than the STN, and the microlesion effect is larger in the STN (Lange et al., 1976; Vasques et al., 2009; Herreras, 2016). Clearly, we cannot directly gauge why power differs across nuclei, and this was not our goal, but exploring this open question may expand our understanding of the basal ganglia and its role in PD.

Next, we observed profound decreases in beta power during movement in the STN and GPi. These findings expand on the functional meaning of beta desynchronization in the STN (Doyle et al., 2005; Lofredi et al., 2019). In contrast to the abundance of STN data, this is one of a few studies exploring such activity in the GPi (Tsang et al., 2012; Tsiokos et al., 2017; Wang et al., 2018).
Processed LFP Signal (Bipolar, 1-100 Hz)

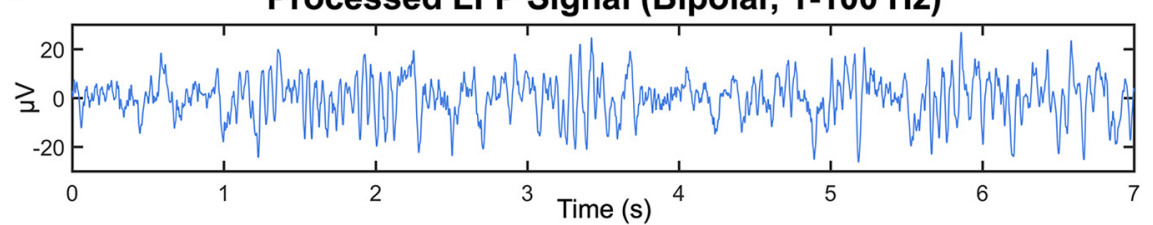

Patient-specific Beta (14-18 Hz)

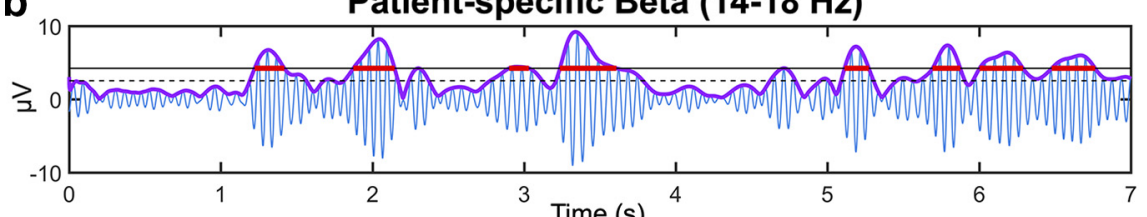

Time (s)

d
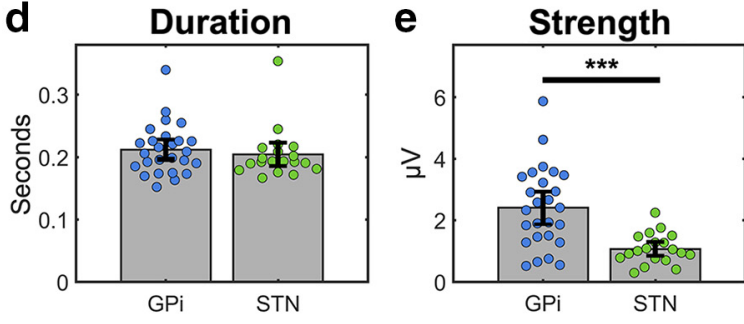

g Patient Level

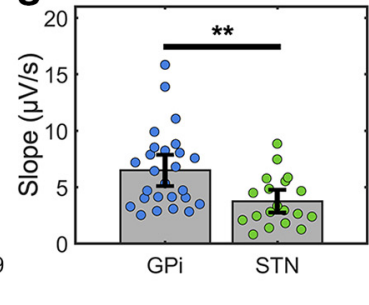

Figure 3. Beta burst properties in the GPi and STN. Bursts were computed using the 75th percentile threshold approach (see Materials and Methods). Blue represents data from GPi participants. Green represents data from STN participants. $\boldsymbol{a}, \boldsymbol{b}$, Example of frequency for this participant with the most beta power desynchronization during movement. The purple line indicates the Hilbert red ine indicate the identified bursts. The average (c) number per second, ( $\boldsymbol{d}$ ) duration, and $(\boldsymbol{e})$ strength of bursts for peticipant. $\boldsymbol{f}$, The relationship between duration and strength across all participants' bursts. $\boldsymbol{g}$, The slope of the fitted line relating the duration and strength of beta bursts at the individual level. Error bars indicate two SEs above and below the mean $(\boldsymbol{c}-\boldsymbol{e}, \boldsymbol{g}) .{ }^{* *} p<0.01,{ }^{* * *} p<0.001$.

This phenomenon in the pallidum does not appear to be specific to PD and has been seen in dystonia (Liu et al., 2006, 2008; Brücke et al., 2008; van Wijk et al., 2017) with smaller magnitude (Wang et al., 2018). We also showed, for the first time, that the degree of beta desynchronization was greater in the GPi than the STN. This could be due to differences in movement mechanics, but button pressing speed was not different between groups. Stronger movement-induced GPi beta desynchronization opens the possibility that movement detection may be more feasible with GPi LFPs.

The ability of beta power to fluctuate in time has been recognized for several decades (Murthy and Fetz, 1992, 1996). Longer bursts are associated with diminished information coding (Brittain and Brown, 2014; Shin et al., 2017). There is a positive relationship between burst duration and amplitude (Torrecillos et al., 2018), the variability of which has not previously been examined across individuals or brain targets. Bursts have been studied in the GPi of individuals with PD in just two studies, which showed that pallidal burst amplitude, but not duration or frequency, differs in dystonia and PD (Wang et al., 2018; PiñaFuentes et al., 2019). In our data, while bursts from the STN and 

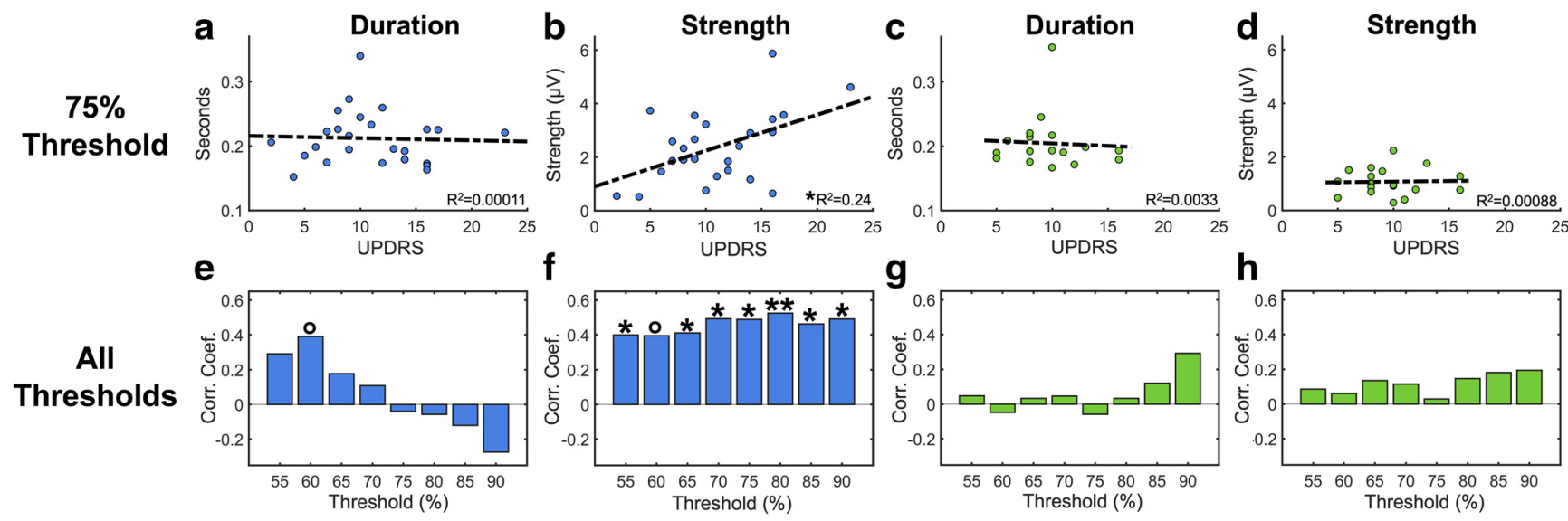

Figure 4. Relationships between bradykinesia-rigidity UPDRS score and burst duration and strength $(\boldsymbol{a}-\boldsymbol{d})$. Blue represents data from GPi participants. Green represents data from STN participants. Bottom, $y$ axis indicates correlation coefficients (Corr. Coef). Because this analysis was exploratory in nature, the multiple correlations (e- $\boldsymbol{h}$ ) do not include $p$ value correction. $\boldsymbol{c}, \boldsymbol{g}$, Insignificant findings are not affected by the STN outlier with a burst duration $\sim 0.35 \mathrm{~s}$. In the GPi, bradykinesia-rigidity duration correlation at threshold $60: R^{2}=0.14, p=0.063$. In the GPi, bradykinesia-rigidity strength correlation from threshold 55\% to 90\%: $R^{2}=0.16, p=0.047 ; R^{2}=0.16, p=0.051 ; R^{2}=0.17, p=0.041 ; R^{2}=0.25, p=0.012 ; R^{2}=0.24, p=0.012 ; R^{2}=$ $0.28, p=0.0067 ; R^{2}=0.22, p=0.017 ; R^{2}=0.23, p=0.016 .{ }^{\circ} p<0.1 .{ }^{*} p<0.05,{ }^{* *} p<0.01$.

GPi showed similar occurrence rates and durations, amplitudes were higher in the GPi. This is also reflected by the fact that the duration-amplitude slope relationship is steeper for GPi bursts. Collectively, this indicates that the burst duration-amplitude relationship may differ across diseases and brain targets.

\section{Clinical applications}

These findings have promising clinical translation. Decreases in beta power occur after levodopa intake or high-frequency stimulation and correlate with improvements in bradykinesia and rigidity, although not tremor (Priori et al., 2004; Doyle et al., 2005; Kühn et al., 2006, 2008, 2009). Future work should test the effect of levodopa, but our finding that off-medication beta power relates to clinical severity, particularly in the GPi, is significant. Prior studies, including ours, have failed to find such a relationship with the STN (Weinberger et al., 2006; Moran et al., 2008; Kühn et al., 2009). In the GPi, we found a correlation between beta power during movement and bradykinesia and rigidity. This is noteworthy because although beta modulates with movement, it suggests the possibility for monitoring clinical impairment across different motor states. Interestingly, the degree of beta desynchronization with movement was not related to motor impairments. Beta desynchronization is recognized as physiologically important for normal motor behavior (Engel and Fries, 2010), and it is unclear whether it is specifically affected in $\mathrm{PD}$, although we can conclude it does not depend on PD symptom severity.

We also examined the relationship between beta burst properties and clinical impairment. We expected to find that prolonged beta bursts were associated with higher bradykinesia-rigidity scores (Tinkhauser et al., 2017a,b). However, the overwhelming lack of a relationship between burst duration and UPDRS in either the GPi or STN calls to question the generalizability of these previously reported effects. Rather than duration, our results suggest that burst strength may hold more consistent clinical relevance. Given that burst duration and strength can be sensitive to increasing thresholds (Tinkhauser et al., 2017b), it was encouraging to see a threshold-resistant relationship between burst strength and clinical impairment in the GPi cohort.

\section{Conclusions and limitations}

We found that beta power modulates more with movement in the GPi and that higher resting beta in the GPi is also reflected in stronger beta bursts, which relate to bradykinesia and rigidity. We are not the first to suggest that the GPi may be a more robust brain target for detection of PD-specific biomarkers (Wang et al., 2018). Chronic recordings with embedded systems are needed to evaluate and compare the efficacy of STN and GPi aDBS for PD.

There are several limitations to this study. First, the decision to implant the STN or GPi was nonrandomized, and there may be important differences across participants that confound our results. Second, it is plausible that, with a larger STN sample, some trends in our data could become more meaningful, although these sample sizes are similar or larger than existing studies of beta power in PD. Third, our data are from acute recordings in the operating room setting, shortly after DBS electrodes are inserted and thus during the lesion effect. Fourth, our recordings are relatively short compared with prior studies; however, in real practice, aDBS decisions will need to take place with even more limited recordings in real time. Last, our findings should be interpreted carefully in the context of prior work because, unlike some studies, we did not specifically exclude participants with tremor predominant PD or those that were lacking visually apparent beta peaks (Tinkhauser et al., 2017b). However, this should improve the generalizability of our results.

\section{References}

Anderson VC, Burchiel KJ, Hogarth P, Favre J, Hammerstad JP (2005) Pallidal vs subthalamic nucleus deep brain stimulation in Parkinson disease. Arch Neurol 62:554-560.

Brittain JS, Brown P (2014) Oscillations and the basal ganglia: motor control and beyond. Neuroimage 85:637-647.

Brown P, Oliviero A, Mazzone P, Insola A, Tonali P, Di Lazzaro V (2001) Dopamine dependency of oscillations between subthalamic nucleus and pallidum in Parkinson's disease. J Neurosci 21:1033-1038.

Brücke C, Kempf F, Kupsch A, Schneider GH, Krauss JK, Aziz T, Yarrow K, Pogosyan A, Brown P, Kühn AA (2008) Movement-related synchronization of gamma activity is lateralized in patients with dystonia. Eur J Neurosci 27:2322-2329.

Deffains M, Iskhakova L, Katabi S, Israel Z, Bergman H (2018) Longer $\beta$ oscillatory episodes reliably identify pathological subthalamic activity in Parkinsonism. Mov Disord 33:1609-1618. 
Doyle LM, Kühn AA, Hariz M, Kupsch A, Schneider GH, Brown P (2005) Levodopa-induced modulation of subthalamic beta oscillations during self-paced movements in patients with Parkinson's disease. Eur J Neurosci 21:1403-1412.

Eisinger RS, Urdaneta ME, Foote KD, Okun MS, Gunduz A (2018) Nonmotor characterization of the basal ganglia: evidence from human and non-human primate electrophysiology. Front Neurosci 12:385.

Eisinger RS, Martinez-Ramirez D, Ramirez-Zamora A, Hess CW, Almeida L, Okun MS, Gunduz A (2019) Parkinson's disease motor subtype changes during 20 years of follow-up. Parkinsonism Relat Disord. Advance online publication. Retrieved May 19, 2019. doi: 10.1016/j. parkreldis.2019.05.024.

Engel AK, Fries P (2010) Beta-band oscillations: signalling the status quo? Curr Opin Neurobiol 20:156-165.

Feingold J, Gibson DJ, DePasquale B, Graybiel AM (2015) Bursts of beta oscillation differentiate postperformance activity in the striatum and motor cortex of monkeys performing movement tasks. Proc Natl Acad Sci U S A 112:13687-13692.

Fischer P, Pogosyan A, Green AL, Aziz TZ, Hyam J, Foltynie T, Limousin P, Zrinzo L, Samuel M, Ashkan K, Da Lio M, De Cecco M, Fornaser A, Brown P, Tan H (2019) Beta synchrony in the cortico-basal ganglia network during regulation of force control on and off dopamine. Neurobiol Dis 127:253-263.

Follett KA, Weaver FM, Stern M, Hur K, Harris CL, Luo P, Marks WJ Jr, Rothlind J, Sagher O, Moy C, Pahwa R, Burchiel K, Hogarth P, Lai EC, Duda JE, Holloway K, Samii A, Horn S, Bronstein JM, Stoner G, et al. (2010) Pallidal versus subthalamic deep-brain stimulation for Parkinson's disease. N Engl J Med 362:2077-2091.

Geng X, Zhang J, Jiang Y, Ashkan K, Foltynie T, Limousin P, Zrinzo L, Green A, Aziz T, Brown P, Wang S (2017) Comparison of oscillatory activity in subthalamic nucleus in Parkinson's disease and dystonia. Neurobiol Dis 98:100-107.

Giannicola G, Marceglia S, Rossi L, Mrakic-Sposta S, Rampini P, Tamma F, Cogiamanian F, Barbieri S, Priori A (2010) The effects of levodopa and ongoing deep brain stimulation on subthalamic beta oscillations in Parkinson's disease. Exp Neurol 226:120-127.

Gilbertson T, Lalo E, Doyle L, Di Lazzaro V, Cioni B, Brown P (2005) Existing motor state is favored at the expense of new movement during 13-35 Hz oscillatory synchrony in the human corticospinal system. J Neurosci 25:7771-7779.

Herreras O (2016) Local field potentials: myths and misunderstandings. Front Neural Circuits 10:101.

Hirschmann J, Schoffelen JM, Schnitzler A, van Gerven MA (2017) Parkinsonian rest tremor can be detected accurately based on neuronal oscillations recorded from the subthalamic nucleus. Clin Neurophysiol 128: 2029-2036.

Hsu YT, Lai HY, Chang YC, Chiou SM, Lu MK, Lin YC, Liu YL, Chen CC, Huang HC, Chien TF, Lin SZ, Chen YY, Tsai CH (2012) The role of the sub-thalamic nucleus in the preparation of volitional movement termination in Parkinson's disease. Exp Neurol 233:253-263.

Jimenez-Shahed J, Telkes I, Viswanathan A, Ince NF (2016) GPi oscillatory activity differentiates tics from the resting state, voluntary movements, and the unmedicated Parkinsonian state. Front Neurosci 10:436.

Kühn AA, Williams D, Kupsch A, Limousin P, Hariz M, Schneider GH, Yarrow K, Brown P (2004) Event-related beta desynchronization in human subthalamic nucleus correlates with motor performance. Brain 127: 735-746.

Kühn AA, Trottenberg T, Kivi A, Kupsch A, Schneider GH, Brown P (2005) The relationship between local field potential and neuronal discharge in the subthalamic nucleus of patients with Parkinson's disease. Exp Neurol 194:212-220.

Kühn AA, Kupsch A, Schneider GH, Brown P (2006) Reduction in subthalamic 8-35 Hz oscillatory activity correlates with clinical improvement in Parkinson's disease. Eur J Neurosci 23:1956-1960.

Kühn AA, Kempf F, Brücke C, Gaynor Doyle L, Martinez-Torres I, Pogosyan A, Trottenberg T, Kupsch A, Schneider GH, Hariz MI, Vandenberghe W, Nuttin B, Brown P (2008) High-frequency stimulation of the subthalamic nucleus suppresses oscillatory $\beta$ activity in patients with Parkinson's disease in parallel with improvement in motor performance. J Neurosci 28:6165-6173.

Kühn AA, Tsui A, Aziz T, Ray N, Brücke C, Kupsch A, Schneider GH, Brown P (2009) Pathological synchronisation in the subthalamic nucleus of pa- tients with Parkinson's disease relates to both bradykinesia and rigidity. Exp Neurol 215:380-387.

Lange H, Thorner G, Hopf A (1976) [Morphometric-statistical structure analysis of human striatum, pallidum and nucleus su-thalamicus: III. Nucleus subthalamicus]. J Hirnforsch 17:31-41.

Leventhal DK, Gage GJ, Schmidt R, Pettibone JR, Case AC, Berke JD (2012) Basal ganglia beta oscillations accompany cue utilization. Neuron 73:523-536

Little S, Pogosyan A, Neal S, Zavala B, Zrinzo L, Hariz M, Foltynie T, Limou$\sin$ P, Ashkan K, FitzGerald J, Green AL, Aziz TZ, Brown P (2013) Adaptive deep brain stimulation in advanced Parkinson disease. Ann Neurol $74: 449-457$.

Little S, Beudel M, Zrinzo L, Foltynie T, Limousin P, Hariz M, Neal S, Cheeran B, Cagnan H, Gratwicke J, Aziz TZ, Pogosyan A, Brown P (2016a) Bilateral adaptive deep brain stimulation is effective in Parkinson's disease. J Neurol Neurosurg Psychiatry 87:717-721.

Little S, Tripoliti E, Beudel M, Pogosyan A, Cagnan H, Herz D, Bestmann S, Aziz T, Cheeran B, Zrinzo L, Hariz M, Hyam J, Limousin P, Foltynie T, Brown P (2016b) Adaptive deep brain stimulation for Parkinson's disease demonstrates reduced speech side effects compared to conventional stimulation in the acute setting. J Neurol Neurosurg Psychiatry 87: 1388-1389.

Liu X, Yianni J, Wang S, Bain PG, Stein JF, Aziz TZ (2006) Different mechanisms may generate sustained hypertonic and rhythmic bursting muscle activity in idiopathic dystonia. Exp Neurol 198:204-213.

Liu X, Wang S, Yianni J, Nandi D, Bain PG, Gregory R, Stein JF, Aziz TZ (2008) The sensory and motor representation of synchronized oscillations in the globus pallidus in patients with primary dystonia. Brain 131: 1562-1573.

Lofredi R, Tan H, Neumann WJ, Yeh CH, Schneider GH, Kühn AA, Brown P (2019) Beta bursts during continuous movements accompany the velocity decrement in Parkinson's disease patients. Neurobiol Dis 127:462471.

Moran A, Bergman H, Israel Z, Bar-Gad I (2008) Subthalamic nucleus functional organization revealed by Parkinsonian neuronal oscillations and synchrony. Brain 131:3395-3409.

Murthy VN, Fetz EE (1992) Coherent 25- to 35-Hz oscillations in the sensorimotor cortex of awake behaving monkeys. Proc Natl Acad Sci U S A 89:5670-5674.

Murthy VN, Fetz EE (1996) Oscillatory activity in sensorimotor cortex of awake monkeys: synchronization of local field potentials and relation to behavior. J Neurophysiol 76:3949-3967.

Okun MS, Fernandez HH, Wu SS, Kirsch-Darrow L, Bowers D, Bova F, Suelter M, Jacobson CE 4th, Wang X, Gordon CW Jr, Zeilman P, Romrell J, Martin P, Ward H, Rodriguez RL, Foote KD (2009) Cognition and mood in Parkinson's disease in subthalamic nucleus versus globus pallidus interna deep brain stimulation: the COMPARE trial. Ann Neurol 65:586-595.

Okun MS, Ramirez-Zamora A, Foote KD (2018) Neuromedicine service and science hub model. JAMA Neurol 75:271-272.

Piña-Fuentes DP, Little S, Oterdoom M, Neal S, Pogosyan A, Tijssen MA, van Laar T, Brown P, van Dijk JM, Beudel M (2017) Adaptive DBS in a Parkinson's patient with chronically implanted DBS: a proof of principle. Mov Disord 74:449.

Piña-Fuentes DP, van Zijl JC, van Dijk JM, Little S, Tinkhauser G, Oterdoom DL, Tijssen MA, Beudel M (2019) The characteristics of pallidal lowfrequency and beta bursts could help implementing adaptive brain stimulation in the Parkinsonian and dystonic internal globus pallidus. Neurobiol Dis 121:47-57.

Priori A, Foffani G, Pesenti A, Tamma F, Bianchi A, Pellegrini M, Locatelli M, Moxon K, Villani RM (2004) Rhythm-specific pharmacological modulation of subthalamic activity in Parkinson's disease. Exp Neurol 189: 369-379.

Ramirez-Zamora AR, Ostrem JL (2018) Globus pallidus interna or subthalamic nucleus deep brain stimulation for Parkinson disease: a review. JAMA Neurol 75:367-372.

Ray NJ, Jenkinson N, Wang S, Holland P, Brittain JS, Joint C, Stein JF, Aziz T (2008) Local field potential beta activity in the subthalamic nucleus of patients with Parkinson's disease is associated with improvements in bradykinesia after dopamine and deep brain stimulation. Exp Neurol 213: $108-113$

Rosa M, Arlotti M, Ardolino G, Cogiamanian F, Marceglia S, Di Fonzo A, 
Cortese F, Rampini PM, Priori A (2015) Adaptive deep brain stimulation in a freely moving Parkinsonian patient. Mov Disord 30:1003-1005.

Rosanova M, Casali A, Bellina V, Resta F, Mariotti M, Massimini M (2009) Natural frequencies of human corticothalamic circuits. J Neurosci 29:7679-7685.

Schalk G, McFarland DJ, Hinterberger T, Birbaumer N, Wolpaw JR (2004) BCI2000: a general-purpose brain-computer interface (BCI) system. IEEE Trans Biomed Eng 51:1034-1043.

Shin H, Law R, Tsutsui S, Moore CI, Jones SR (2017) The rate of transient beta frequency events predicts behavior across tasks and species. Elife 6:572.

Silberstein P, Kühn AA, Kupsch A, Trottenberg T, Krauss JK, Wöhrle JC, Mazzone P, Insola A, Di Lazzaro V, Oliviero A, Aziz T, Brown P (2003) Patterning of globus pallidus local field potentials differs between Parkinson's disease and dystonia. Brain 126:2597-2608.

Singh A, Bötzel K (2013) Globus pallidus internus oscillatory activity is related to movement speed. Eur J Neurosci 38:3644-3649.

Stein E, Bar-Gad I (2013) $\beta$ oscillations in the cortico-basal ganglia loop during Parkinsonism. Exp Neurol 245:52-59.

Sudhyadhom A, Okun MS, Foote KD, Rahman M, Bova FJ (2012) A threedimensional deformable brain atlas for DBS targeting: I. Methodology for atlas creation and artifact reduction. Open Neuroimag J 6:92-98.

Tinkhauser G, Pogosyan A, Little S, Beudel M, Herz DM, Tan H, Brown P (2017a) The modulatory effect of adaptive deep brain stimulation on beta bursts in Parkinson's disease. Brain 140:1053-1067.

Tinkhauser G, Pogosyan A, Tan H, Herz DM, Kühn AA, Brown P (2017b) Beta burst dynamics in Parkinson's disease OFF and ON dopaminergic medication. Brain 140:2968-2981.

Tinkhauser G, Torrecillos F, Duclos Y, Tan H, Pogosyan A, Fischer P, Carron R, Welter ML, Karachi C, Vandenberghe W, Nuttin B, Witjas T, Régis J, Azulay JP, Eusebio A, Brown P (2018) Beta burst coupling across the motor circuit in Parkinson's disease. Neurobiol Dis 117:217-225.

Torrecillos F, Tinkhauser G, Fischer P, Green AL, Aziz TZ, Foltynie T, Limousin P, Zrinzo L, Ashkan K, Brown P, Tan H (2018) Modulation of beta bursts in the subthalamic nucleus predicts motor performance. J Neurosci 38:1314-1318.

Tsang EW, Hamani C, Moro E, Mazzella F, Lozano AM, Hodaie M, Yeh IJ, Chen R (2012) Movement related potentials and oscillatory activities in the human internal globus pallidus during voluntary movements. J Neurol Neurosurg Psychiatry 83:91-97.

Tsiokos C, Malekmohammadi M, AuYong N, Pouratian N (2017) Pallidal low $\beta$-low $\gamma$ phase-amplitude coupling inversely correlates with Parkinson disease symptoms. Clin Neurophysiol 128:2165-2178.

van Wijk BC, Neumann WJ, Schneider GH, Sander TH, Litvak V, Kühn AA (2017) Low-beta cortico-pallidal coherence decreases during movement and correlates with overall reaction time. Neuroimage 159:1-8.

Vasques X, Cif L, Hess O, Gavarini S, Mennessier G, Coubes P (2009) Prognostic value of globus pallidus internus volume in primary dystonia treated by deep brain stimulation. J Neurosurg 110:220-228.

Velisar A, Syrkin-Nikolau J, Blumenfeld Z, Trager MH, Afzal MF, Prabhakar V, Bronte-Stewart H (2019) Dual threshold neural closed loop deep brain stimulation in Parkinson disease patients. Brain Stimul 12:868876.

Wang DD, de Hemptinne C, Miocinovic S, Ostrem JL, Galifianakis NB, San Luciano M, Starr PA (2018) Pallidal deep-brain stimulation disrupts pallidal beta oscillations and coherence with primary motor cortex in Parkinson's disease. J Neurosci 38:4556-4568.

Weinberger M, Mahant N, Hutchison WD, Lozano AM, Moro E, Hodaie M, Lang AE, Dostrovsky JO (2006) Beta oscillatory activity in the subthalamic nucleus and its relation to dopaminergic response in Parkinson's disease. J Neurophysiol 96:3248-3256.

Weinberger M, Hutchison WD, Alavi M, Hodaie M, Lozano AM, Moro E, Dostrovsky JO (2012) Oscillatory activity in the globus pallidus internus: comparison between Parkinson's disease and dystonia. Clin Neurophysiol 123:358-368.

Wingeier B, Tcheng T, Koop MM, Hill BC, Heit G, Bronte-Stewart HM (2006) Intra-operative STN DBS attenuates the prominent beta rhythm in the STN in Parkinson's disease. Exp Neurol 197:244-251.

Wong J, Gunduz A, Shute J, Eisinger R, Cernera S, Ho KW, Martinez-Ramirez D, Almeida L, Wilson CA, Okun MS, Hess CW (2018) Longitudinal follow-up of impedance drift in deep brain stimulation cases. Tremor Other Hyperkinet Mov (N Y) 8:542.

Yang AI, Vanegas N, Lungu C, Zaghloul KA (2014) Beta-coupled highfrequency activity and beta-locked neuronal spiking in the subthalamic nucleus of Parkinson's disease. J Neurosci 34:12816-12827. 